\title{
Exploration of the Thinking Model of APP Interactive Experience Design Based on "Internet +"
}

\author{
Yi Yang \\ Hubei Academy of Fine Arts, Wuhan, Hubei, China, 430020
}

Keywords: Exploration, Thinking Model, APP, Interactive Experience Design, "Internet +"

\begin{abstract}
On the basis of analyzing the idea of "Internet +", this paper combines the thinking mode of "Internet +" with APP interactive experience design, and puts forward the design model of APP interactive experience based on "Internet $+"$. Then the overall model structure of the model is analyzed, and the core position of the user is emphasized. Based on the user experience, the demand analysis of the interactive design model, the design of APP and the user experience are analyzed. It is hoped to provide theoretical support for the design and development of APP for smart phones through the construction of the design interaction model of APP interactive experience based on "Internet +".
\end{abstract}

\section{Introduction}

With the increasingly mature communication technology, the popularity of $3 G$ networks, the Internet IT industry to a third peak - "the mobile Internet boom." Mobile Internet equipment will undoubtedly become the darling of this era. More well-known application store has Apple's APP Store, Google's Google Play Store, An Zhi market, as well as BlackBerry users BlackBerry APP World, Microsoft's Marketplace and so on. APP's digital technology can establish a connection with the user and interact, so that users can touch or slide the interface to the autonomy of browsing information. And the new media interface to show the user is the traditional physical media in the paper, graphics, information digital performance, this digital performance to expand the layout of the connotation and extension, and layout design provides a new opportunity.

With the implementation of the national "Internet +" strategy, the mobile Internet, represented by smart phone applications, is playing an increasingly important role in economic development. With the national "Internet +" strategic thinking of the deepening, all walks of life have "touch the net", the use of smart phones APP, We-Chat public account, mobile network mall and other mobile applications and users to achieve wireless Internet. "Internet +", from the perspective of Marxist philosophy, is a philosophical embodiment of the relationship between thinking, reflecting the Internet and all walks of life links and integration. From the user experience point of view, "Internet $+"$ is the user experience for the product experience to the social, emotional experience of the transition. From the perspective of design innovation, "Internet +" is the way of innovative thinking, reflecting the development of design thinking.

At present, in the design of smart phone APP, the design thinking of continuous improvement and development, from the function as the center to the development of the user-centered, from the development of the "waterfall" design to "agile" design, embodies the APP design from Low to high, from simple to constantly perfect design process. APP design practice proved that the user experience design as the basis, emphasizing integration, cross-border and innovation, can effectively improve the design level. To this end, this article based on the "Internet + " core ideas as a guide, based on the user experience to build intelligent mobile APP interactive experience design thinking model, designed to user experience design, to explore APP design thinking rules for smart phone APP design And provide theoretical support for development.

\section{The Concept Definition of "Internet +"}

Chinese Academy of Engineering, China Internet Association, said Wu Hequan, "Internet +" is the Internet to enhance the function and application development, is a new stage of the Internet. Li 
Yanhong that "Internet + " means that the Internet and other traditional industries combined model. Is not "Internet" and "Internet +", it represents a new economic form, that is, give full play to the Internet in the production of social factors in the optimization and integration of the role of the Internet innovation in the depth of integration of social and economic fields, Enhance the innovation and productivity of the real economy, and form a new form of economic development with the Internet as the infrastructure and the realization of the tools. It is worth noting and need to emphasize that, although the "Internet +" is "Internet + all traditional industries", but it is not a simple sum of the two. "Internet +" to promote a series of magical "chemical reaction", which is like electricity in the second industrial revolution so that many industries have changed dramatically. The future of the Internet will be the same as electricity, as a production tool, to bring profound changes in various industries. In other words, "Internet + " to the traditional industries to further penetrate and transform the Internet.

\section{The Production of APP}

APP appeared in the earliest to the phone candy era NOKIA mobile phone built-in Snake game. At that time the phone is a monochrome display, relying on the navigation button to operate, so the game is relatively simple, but it gives the user's entertainment cannot be ignored, and the nature of the game makes the current market appears APP still game the Lord. Because it is built in the phone before the factory built-in mobile phone, the user can not modify their own, these games are mostly considered part of the mobile phone function, but from today's point of view of APP can be seen when the game like Snake Both the current prototype of APP.

With the mobile device into the functional era, APP's development has entered a new platform. JAVA and other programming technology development and popularization, there have been many available for users to install, uninstall the application, which is the main game entertainment category, which formed the initial APP. At the same time, GPRS promotion, making the phone connected with the Internet, the Internet began to produce APP making the initial entertainment-oriented APP began to information, social, tools and other direction. The emergence of smart phones also contributed to the development of APP, compared to non-smart phones, smart phones have their own operating system, an independent processor, and a larger display screen, prompted the development of APP standardization, smooth operation and Diversification of performance for the design and development to provide convenience.

\section{The Development Trend of APP}

Socialization. Sociality is one of the most frequent nouns in the Internet industry. Facebook, twitter and other social Internet products launched, making the feelings of indifferent between the modern society through the Internet once again produced a communication, and break the geographical, time constraints. The emergence of social products not only for users to build a platform to share life, but also for developers gathered a large number of users, provides a guarantee for its development. Mobile Internet is bound to conform to the development trend of social development, because the original design products are based on the personal computer platform, in the information transmission and operation is not entirely suitable for mobile devices. Blindly the function of Web products copied to the APP is bound to its users to use the impact. This indicates that in the mobile Internet era, social networking will be a new innovation and APP will gradually evolve for mobile devices, new social products.

Localization. Localization is a unique feature of mobile devices, but also mobile Internet unique advantages. In the early stages of development, the characteristics of its localized APP are not obvious enough, but with the community's concern for LBS, LBS will be the direction of the next APP development. For the Internet to identify the illusory and untrue users, LBS is to dispel their concerns of an effective technology, through the wireless network positioning technology, LBS not only can determine the user's location, allowing users to clearly understand their own environment, So that users in a strange environment will not have a sense of frustration, and can provide users 
with the location of the user's surrounding information, so that users get a convenient service. The emergence of LBS not only for the life support class APP provides a new platform for e-commerce, life assistant products have a new direction, but also eliminates the use of some users concerns for APP to win more user groups. It is easy to see that in the future development of APP, the integration with LBS technology is an irresistible trend.

Mobile. APP because of the mobile device, its mobility is no doubt. The mobility here refers to APP can be many of the original must be carried out at a specific location of the event, can be followed by the user at any point in the implementation of the convenience of the user. Mobility gives the user the greatest experience is to cancel the restrictions, to provide users with a free way of life. Human beings are free animals, when the original life on the fast-paced human beings to impose a lot of unnecessary restrictions on the human psychological and physical trauma is evident. In order to improve the quality of life, the liberation of the user's physical and mental, many APP manufacturers have begun to design and develop some products to meet the user's shopping, payment, ordering and other activities. I believe that soon after, there will be more APP will be users from a specific working environment in the liberation of the user to create a free life.

\section{The APP Interactive Experience Design Thinking Model Based on the "Internet +"}

Take the User Model as the Core. The user model is the core of the APP design phase, which directly affects the success of APP design and development. The closer the user model is to the actual user's use of APP, the more true the user experience. The user model is based on simple and beautiful thinking, socialization and platform thinking based on the model, is the user needs in accordance with the simple and beautiful standards, combined with the use of APP users in the context of the social and communication platform needs to establish. The user cognitive model needs to analyze the cognitive characteristics of the user from the perspective of psychology, and effectively combine the design of the information structure with the design of the user's cognitive model. The user behavior model needs to consider the different users for the smart phone APP application habits and behavior characteristics try to use similarity design for behavior guidance. The user role model is a user model of the extraction and vivid portrayal, for the prototype design provides a user role support and then lay the foundation for the development and testing of APP.

Reflect the Simple and Beautiful Thinking. Users for the smart phone APP expectations, simply the biggest voice of the user. On the basis of user needs analysis, combined with simple thinking to sort out the user needs and induction, to extract the application of the core functions of APP and aesthetic needs, reflecting the "focus, less is more" and "simple is the United States" design. Based on the user's visual information on the basis of the aesthetic thinking is that every APP designer first to consider the issue, to achieve APP simple, barrier-free use of APP design is the inherent requirements. Simple and beautiful thinking, both the icon, color, layout and interface simple and beautiful, but also includes the interactive process of simple and convenient and interactive way of human design, both static interface information in line with the needs of users, but also requires dynamic display rhythm And rhythm in line with the user's expectations. Mito Xiu show APP is to seize the user for the pursuit of beauty, through a simple interface and practical features to meet the needs of users beautify the picture.

Reflect the Socialization and Platform Thinking. On the basis of user demand analysis, combined with the socialization and platform thinking to refine the user needs and combine to consider the app application of social and user experience, trying to use the APP application for users to create a platform for communication. Socialization and platform thinking embodies the sentiment of life, especially for a certain user base APP. When users use Taobao to shop, check the buyer's comments and the seller's level to become an important step before the user purchase, the buyer's evaluation of goods or services for the user to provide a reputation to support the purchase. Taobao community for the user to share shopping experience and exchange ideas to provide a topic platform, the buyer's real shopping experience, enthusiastic reply for the many Amoy Friends shopping experience to increase the emotional interaction, can be described as emotional sublimation and enjoyment. And when the user presses the phone's screenshots button, Taobao 
automatically pop up the sharing interface can be described as an effective tracking of user behavior, greatly improving the Taobao emotional score in the user's mind, so that Taobao users indispensable mobile shopping platform.

Take Fusion and Cross-Border Thinking As the Breakthrough. "Internet +" era is a fusion, innovation and cross-border era, based on large data thinking, to integration and cross-border thinking APP design and related industries, services and needs together to achieve the APP design breakthrough. Combined with the user experience, the use of mobile phone based on the location of the service features for users to recommend related services, so that APP other applications into the portal, to create APP platform effect. Baidu glutinous rice in the user location based on the user to provide business services at the same time information, but also Baidu map service link to the application, the user arrived at the location where the business mileage and time data provided to the user, and provide route navigation services. In addition to providing VIP privilege and content privilege for VIP members, VIP members also offer exclusive services such as privilege privilege and life privilege. VIP members can obtain coupons in their malls, Goods, financial management, etc., this integration and cross-border thinking, it is the "Internet + " mode of thinking in the video APP and life services, financial integration in the embodiment.

\section{Conclusion}

Mobile Internet era is the mobile intelligence era with the prosperity of APP and in-depth user experience. When users use the APP, the user experience and share the feelings and get happy with the process. In the era of "Internet +", the design of thinking model based on "Internet $+"$ is designed for the design of smart-phone APP, it is very necessary both from the point of view of user experience design or from interactive design method.

\section{Acknowledgements}

Fund Project: Mobile Device Information Organizational and Visual Design Based on Specific Subject

\section{References}

[1] Jia Xinzhang, Li Jingyuan. Research Policy, 2012, Vol. 6 (2014) No 53, p.25-26

[2] Peng Sue, Wang Yunhui, Wang Qunyong. Journal of Organizational Change Management, Vol. 12 (2015) No 27, p.74-76

[3] Qian Xiyuan, Jing Jianfen. Journal of Business Research, Vol. 30 (2014) No 19, p.144-145

[4] Wang Kuailiang. Corporate Governance, Vol. 29 (2008) No 27, p.21-23

[5] Zhang Gongxu, Sun Jing. New Quality Management, Vol. 8 (2013) No 27, p.57-60 